\title{
Reflexiones sobre la enseñanza - aprendizaje de lenguas extranjeras en la carrera de Ingeniería Civil
}

\section{Enseñanza aprendizaje de lenguas extranjeras}

\author{
Jaime Walter Cañarte Ávila, Mg. DUIE ${ }^{(1)}$ \\ Ned Quevedo Arnaiz Dr. C. ${ }^{(2)}$ \\ Nemis García Arias Dra. C. (3) \\ (1) Universidad de Camagüey Ignacio Agramonte, Cuba \\ ${ }^{(2)}$ Universidad de Camagüey Ignacio Agramonte, Cuba \\ ${ }^{(3)}$ Universidad de Camagüey Ignacio Agramonte, Cuba
}

Contacto: jacanarte64@ hotmail.com

Receptado: 9 /05/2014 Aceptado: 20 /05/ 2014

\section{Resumen}

Este trabajo investigativo tiene como objetivo reflexionar el tratamiento que se le da a la formación y desarrollo de la competencia comunicativa oral en las condiciones actuales en que se emplean modalidades que combinan lo tutorial y lo presencial en la enseñanza aprendizaje del inglés para la carrera de Ingeniería Civil en el Ecuador, mediante métodos de análisis y síntesis y el análisis de documentos se reflexiona desde posiciones flexibles las posibilidades de desarrollo que esa combinación entre las realidades presenciales y las virtuales ofrecen para el aprendizaje del inglés como lengua extranjera en la formación del profesional.. De ahí que el problema es el restringido tratamiento metodológico en la enseñanza aprendizaje del inglés que obstaculiza el desarrollo de la competencia comunicativa oral para la Ingeniería Civil. Su campo de Acción el Tratamiento a la competencia comunicativa oral del inglés en la modalidad combinada, tutorial y presencial. Se argumenta la importancia del protagonismo del estudiante como indicador de calidad del aprendizaje, que incluye a su vez atención a la diversidad educativa. Criterios que parten de los postulados del enfoque histórico cultural de L. S. Vygotsky, que sirven de guía para realizar propuestas que combinen lo presencial y lo tutorial en la enseñanza y el aprendizaje. Se precisa garantizar la formación y desarrollo de actitudes y valores, y la apropiación de conocimientos y habilidades, comunes y específicos del estudiante, desde el Inglés, como expresión de un enfoque interdisciplinario.

Palabras claves: proceso de enseñanza aprendizaje, formación del profesional, competencia comunicativa en inglés, modalidad presencial y tutorial 


\title{
Reflections on the teaching - learning of foreign languages in Civil Engineering
}

\begin{abstract}
This research work aims at reflecting the treatment given to the formation and development of oral communicative competence under current conditions in which tutorial guidance and faceto-face guidance combine in the learning of English for the Civil Engineering Major. By the using methods of analysis and synthesis and analysis of documents from flexible positions, it is reflected the development possibilities that combination between the face-to-face and virtual realities offer for English learning as a foreign language in the professional. Hence that the problem of the research is restricted methodological approach in the learning of English that hinders the development of oral communication skills for Civil Engineering. Its field of action is the treatment of oral communicative competence in English combined, face- to- face and tutorial guidances, in Civil Engineering. The importance of student leadership as a quality indicator of learning, which in turn includes attention to educational diversity is argued. Criteria are based on the principles of cultural historical approach to L S Vygotsky, that guide to make proposals that combine the face-to- face and tutorial guidances, in teaching and learning.It is necessary to ensure the training and development of attitudes and values, and the appropriation of knowledge and skills, common and specific of the student from the English, as an expression of an interdisciplinary approach.
\end{abstract}

Keywords: teaching and learning process, professional training, communicative competence in English, face-to- face , tutorial guidances

\section{Introducción}

La pedagogía actual trata de fundamentar cómo llegar a los estudiantes de la presente generación de la manera más apropiada para que dominen conocimientos conceptuales, procedimentales y actitudinales para la vida y la profesión, pero también que aprendan una lengua extranjera para la comunicación internacional, como expresión de cultura y modo de actualizar o educar a los profesionales en formación en el Ecuador, técnica o humanísticamente.

Ante los retos actuales, hay investigaciones que estudian la semipresencialidad que ofrece un nuevo marco de referencia para una implementación educativa diferente a lo tradicional; no obstante, aún no se logran los patrones de excelencia necesarios ni se han explicado las 
mejores vías para incorporar el aprendizaje presencial en inglés con las actividades semipresenciales.

Por tanto, se necesita de un verdadero deseo de aprender y practicar, mucho autocontrol y auto exigencia para favorecer el proceso de aprendizaje desde la independencia cognoscitiva, y el empleo de los medios de enseñanza que resulten de mayor significación en el desarrollo del conocimiento y para que contribuyan al equilibrio entre educación y formación, sobre todo en carreras que demandan de independencia cognoscitiva como la Ingeniería Civil.

Es por ello que en este trabajo de investigación el objetivo está dirigido a analizar el tratamiento que se le da a la formación y desarrollo de la competencia comunicativa oral en las condiciones actuales en que se emplean modalidades combinadas entre lo tutorial, fuera del aula, y lo presencial, que se caracteriza por la presencia física de los alumnos y el profesor en el aula de clase, en la enseñanza aprendizaje del inglés para la carrera de Ingeniería Civil.

La modalidad tutorial es la atención personalizada que realiza un académico denominado Profesor tutor, dirigida a apoyar a los estudiantes que así lo requieran, para fortalecer sus procesos de aprendizaje relacionados con los saberes teóricos y heurísticos de las experiencias educativas.

\section{Desarrollo}

En el Ecuador el proceso de enseñanza del idioma inglés ha ido cambiando a través del tiempo, anteriormente, no se le daba mucha importancia a esta asignatura. El inglés era impartido en los establecimientos educativos públicos durante pocas horas con métodos donde imperaba el tradicionalismo y que atendían lo estructural-conductista de la lengua. A los estudiantes se les hacía difícil el aprendizaje del idioma y muchas veces se tornaba aburrido y no motivante sobre todo en estudiantes que necesitan ser creativos y comunicarse.

El uso de métodos activos bajo el enfoque comunicativo en la enseñanza de idiomas permitió la inclusión de los medios de enseñanza como la grabadora, textos de apoyo, laboratorios y videos entre otros, que facilitaron el cambio en la enseñanza y el aprendizaje del inglés, pero estos medios tecnológicos implicaron cambios conceptuales que aun hoy surgen y se desarrollan vertiginosamente.

En la carrera de Ingeniería Civil, la aplicación de métodos activos e integradores, con ayuda de la tecnología, para la enseñanza aprendizaje del idioma inglés propende al desarrollo de habilidades orales y escritas, para lograr comprensión de la información y comunicarse con claridad y eficacia con hablantes de este idioma. Este énfasis en el aprendizaje del inglés 
permite garantizar un perfil de egreso al estudiante con que demuestra su alta competencia profesional y formación técnica, con que alcanza a competir con los parámetros del graduado de otras universidades.

En este ámbito educativo de la escuela como institución, y de la enseñanza-aprendizaje presencial, los procesos que ocurren son complejos. Todo conocimiento nuevo genera varios procesos entre el profesor y el alumno que conlleva a utilizar un método adecuado de interacción comunicativa entre ellos, lo que, a su vez, permite la realización de la enseñanza y el aprendizaje donde ambos se complementan.

La enseñanza se dirige a que el alumno desarrolle su intelecto, sus actitudes y sentimientos, pero este proceso individual ocurre gracias a un intercambio social que comienza por la acción del profesor.

Se enfatiza que para la enseñanza, el aprendizaje es lo más importante, en tanto todas las situaciones de enseñanza acaban convirtiéndose en situaciones de aprendizaje para el alumno, más cuando se trata de prepararse en una lengua extranjera que todo se convierte en interacción. Así, la experiencia como docente de estos investigadores indica que muy a menudo se produce una confusión o identificación entre aprendizaje y enseñanza, cuando enseñanza no es aprendizaje si realmente no se llega al alumno y se justifica y se evalúa todo proceder pedagógico bajo los resultados que obtiene el alumno.

Para Asencio (1986) y otros, la enseñanza se ocupa de atender los procesos de aprendizaje. El alumno logra precisamente su aprendizaje porque está mediado en ese contexto de enseñanza y aprendizaje en que los conocimientos que no puede lograr por sí mismo se le facilitan con la ayuda del profesor. Es decir, la enseñanza es una mediación entre el alumno y lo que tiene que aprender. Una mediación que casi siempre se realiza de forma presencial pero que en los momentos actuales ocurren situaciones de comunicación y de aprendizaje en entornos virtuales que hay que atender.

Consecuentemente, el proceso de aprendizaje será eficiente si el alumno aprende bajo sus decisiones y hasta espontáneamente. Por eso entonces, cuando se enseña se debe de logar que se aprenda y viceversa en cualquier escenario, ora enseñanza y aprendizaje presencial ora enseñanza y aprendizaje virtual. Los dos términos están íntimamente ligados dentro del 
proceso, puesto que, sin enseñanza no puede haber aprendizaje, y sin mediación entre ellos no hay proceso formal.

Un alumno aprende bien, no porque actúe solo y el maestro no le exponga el material planificadamente, sino que sabe pensar, se le enseña a pensar, el maestro le facilita el acto de razonar, le sirve de modelo, le orienta cómo hacerlo; si no tiene experiencia en este sentido, le facilita situaciones de aprendizaje, lo guía. En definitiva, enseñar es una cosa y aprender es otra, pero de su interrelación dialéctica depende el éxito del proceso de enseñanza aprendizaje del inglés y de cualquier otra lengua porque se trata de interacción comunicativa.

El proceso de enseñanza y aprendizaje es un proceso de comunicación, de socialización; donde el profesor comunica - expone - organiza - facilita los contenidos científico - históricos - sociales a los alumnos, y éstos, además de comunicarse con el profesor, lo hacen entre sí y con la comunidad. Este proceso lo llama proceso docente que es un proceso de intercomunicación entre el grupo humano. Los autores de este trabajo le agregarían a esta definición que este proceso de enseñanza aprendizaje permite el crecimiento cultural humano por su incidencia directa e indirecta en el desarrollo del pensamiento que posibilita todos esos procesos mencionados. Álvarez de Zayas, (1999).

El proceso como sistema integrado, constituye en el contexto escolar un proceso de interacción e intercomunicación de varios sujetos, en el cual el maestro ocupa un lugar de gran importancia como pedagogo porque lo organiza y conduce, pero en el que no se logran resultados positivos sin el protagonismo, la actitud y la motivación del alumno, el proceso con todos sus componentes y dimensiones, condiciona las posibilidades de conocer, comprender y formarse como personalidad. Los elementos conceptuales básicos del aprendizaje y la enseñanza, con su estrecha relación, donde el educador debe dirigir los procesos cognitivos, afectivos y volitivos que se deben aprehender conformando las estrategias de enseñanza y aprendizaje.

Como se conoce el hombre es un ser eminentemente social, no crece aislado, sino bajo el influjo de los demás y está en constante reacción por esa influencia. La enseñanza resulta así, no solo un deber, sino un efecto del desarrollo social humano, puesto que es el medio con que la sociedad preserva los saberes con que ella misma se desarrolla. Por tanto, como existe el deber de la enseñanza, también, existe el derecho de que se faciliten los medios para 
Rev. SINAPSIS, Vol. 4, No 1, Junio 2014

ISSN $1390-7832$

adquirirla, para facilitar estos medios se encuentran como principales protagonistas el Estado o entidades privadas, quienes facilitan los medios, y los individuos, que son quienes han de adquirir todos los conocimientos necesarios en pos de su logro personal y el engrandecimiento de la sociedad. Por ello, en las condiciones actuales, la tendencia de la enseñanza se dirige hacia la disminución del contenido puramente teórico, más bien se complementa con la práctica y asimila varios métodos que facilitan al alumno su aprendizaje.

En la enseñanza aprendizaje de lenguas extranjeras se hace necesario que los conocimientos conceptuales, procedimentales y actitudinales perduren para que se formen y desarrollen las habilidades y hábitos con que el usuario de ese idioma puede ser competente y desarrollar su competencia comunicativa en cualquier contexto en que este interactúe en ese idioma.

Según H. D. Brown (2000).

Aprender una segunda lengua es prolongado y complejo. Todo en la persona se debate en una lucha por alcanzar confines más allá de la primera lengua en una nueva lengua, nueva cultura, nueva forma de pensar, sentir y actuar. Un comprometimiento total, una involucración total y una total respuesta física, intelectual y emocional son necesarios para enviar y recibir mensajes con éxito en la lengua extranjera... aprender una lengua no es un conjunto de pasos fáciles que pueden ser programados... el aprendizaje exitoso de una lengua extranjera no se logra solamente enmarcado en las paredes del aula.

Esto indica que lo presencial ha de ser acompañado de otras formas no presenciales y hasta virtuales de interacción que de manera integral atiendan la esencia transformadora que implica la enseñanza y el aprendizaje.

En el proceso de enseñanza aprendizaje hay que darle la posibilidad al estudiante de que pueda desarrollar sus potencialidades de acuerdo con sus características individuales hasta lograr que gane la independencia requerida para la dirección de sus propias actividades de aprendizaje.

El proceso de enseñanza aprendizaje del inglés en la Universidad, no solamente debe tomar en cuenta la existencia de un diseño curricular bien concebido, bien estructurado y con la fundamentación científica requerida, sino fundamentalmente su desarrollo, que además de 
considerar el qué y el cuándo enseñar, particularice en quién enseña y quién aprende atendiendo cuidadosamente el cómo enseñar para aprender, o sea, que se facilite la autonomía del aprendizaje, aspecto este tratado en varias investigaciones recientes (Rodríguez, 2008 y García, 2013) y que se convierte en un propósito de este estudio que tiene la intención de incidir en la preparación para interactuar educativamente desde las redes sociales. La implicación de los sujetos del proceso reafirma la aspiración de lograr el papel protagónico del estudiante en su aprendizaje y compromete la actuación del docente en tal dirección, sin desconocimiento del rol decisivo del primero, y del valor de las relaciones mutuas entre ellos y entre los miembros del grupo, así como de la influencia de factores diversos, de uno y otro lado.

Los autores de este trabajo concuerdan con Valenzuela (2007) cuando planteó que hoy: "La Universidad debe impulsar la optimización de sus métodos, capacitar a sus docentes y generar mecanismos de actualización, a fin de articularlos como un medio de apoyo a las actividades previamente planificadas" Así el docente impulsará además la planificación y control del aprendizaje del alumno y dará herramientas para que este cada vez logre mayores niveles de autonomía en su realización, pues hoy prevalece en los cambios educativos el uso de la tecnología y con ellos nuevas necesidades de aprendizaje autónomo.

Visto a través del uso de la tecnología el aprendizaje del inglés ha sido totalmente modificado en las instituciones educativas. Un primer momento transformador ocurrió en la enseñanza aprendizaje de idiomas, alrededor de los años setenta del siglo pasado, y está relacionado con la asunción de la visión amplia y abarcadora del concepto de enfoque comunicativo, con el que el cambio no se limitó a lo cuantitativo, sino a lo cualitativo, de una concepción estructural a una concepción funcional del idioma con intercambios directos entre los usuarios de la lengua. Un segundo momento se debe a la revolución marcada por la telemática, la robótica y las autopistas de la comunicación que conducen a una cibercultura que inexorablemente acorta distancias y tiempo con intercambios virtuales.

Desde ese primer momento, el enfoque comunicativo exige el cumplimiento del rol protagónico al estudiante para la apropiación de la lengua, visto desde la óptica de un proceso de enseñanza-aprendizaje desarrollador, el cual plantea al aprendizaje como la apropiación activa y creadora de la cultura por el sujeto que a su vez alcanza el auto perfeccionamiento constante de su autonomía y autodeterminación en íntima relación con los procesos de 
Rev. SINAPSIS, Vol. 4, No 1, Junio 2014

ISSN $1390-7832$

socialización, tal como expresan Doris Castellanos ( 2002 y 2005), y otros. Apreciaciones que son abordadas por varios autores más desde diferentes aristas de estudio como F. González (2004), F. Addine (2002), y otros, M. Silvestre y P. Rico (2003), F. Addine, A. González y S Recarey. (2004), M. Martínez (2007), M. Alfonso (2010) quienes además, enfatizan que se debe lograr un aprendizaje vivencial - experiencial, con el cual se estimule la reflexión, no sólo de lo cognitivo, sino también de lo afectivo y la unidad de ambos elementos como sistema.

El profesor de inglés diariamente debe enfrentar al reto de búsqueda de estrategias de enseñanza que propicien aprendizajes de la lengua inglesa dirigidos al crecimiento integral de sus estudiantes y de su competencia en el idioma extranjero a partir de la apropiación de los conocimientos y habilidades de forma activa y transformadora y en estrecha vinculación con actitudes y valores. El enfoque comunicativo les permite pues ese desarrollo de la competencia comunicativa que implica la expresión, interpretación y negociación de significados en la interacción entre las personas o entre estas y un texto en lengua extranjera y su crecimiento como sujeto social que se educa.

Del desarrollo de la competencia comunicativa en inglés depende la posibilidad del estudiante de comunicarse en esa lengua extranjera satisfactoriamente, o sea desarrollar todas las habilidades básicas de la lengua, entre ellas hablar, la cual tiene gran relevancia en el mundo actual. La expresión oral es la forma de expresar sin barreras lo que se piensa para comunicarse oralmente con efectividad y llevar la información a otro u otros mediante un canal primario de interacción. La expresión oral sirve como instrumento para comunicar sobre procesos $\mathrm{u}$ objetos externos a un sujeto y por tanto este necesita atender todas las posibilidades expresivas tanto verbales como no verbales, las cuales en una lengua que se aprende fuera de su contexto natural implica intencionalidad por parte del que enseña para que así lo acepte el que aprende y lo use. Por tanto, la importancia del aprendizaje de lo no verbal como complemento de la oralidad es crucial. Recuérdese que A. Mehrabian, un psicólogo social, midió la efectividad de los mensajes comunicativos en condiciones de ambigüedad y su resultado en porcientos fue aproximadamente un $7 \%$ verbal (palabras), un $38 \%$ vocal (tono de voz, ritmo, etc.) y en un 55\% no verbal (gestos, posturas y otros). (Allende, 2011) 
La inclusión de la enseñanza de lenguas extranjeras con fines específicos en los planes de estudio de los colegios y universidades ecuatorianas ha requerido de un gran esfuerzo. A pesar de que se ha estado trabajando por el desarrollo de la expresión oral, ésta todavía continúa subordinada a la lectura, además que no se le dedica el tiempo requerido. La destreza oral puede desarrollarse partiendo del resto de las habilidades del idioma. Así, sobre la base de un texto escrito, se pueden realizar resúmenes orales acerca de lo leído, lo mismo puede hacerse después que el estudiante escucha o escribe sobre un tema determinado para que opine y debata.

Pero en el logro comunicativo del inglés continúan incidiendo elementos totalmente atendibles desde la práctica pedagógica. En este sentido Y. González frece diferentes concepciones y elementos que inciden sobre la situación actual, que no es del todo favorable para atender balanceadamente el desarrollo de las habilidades en idioma extranjero.

1. Énfasis en la repetición oral en lugar de la comunicación oral; una tendencia a hacer preguntas que requieren respuestas cortas en lugar de inducir a la conversación; el uso de ejercicios de repetición oral mecánica con tonalidades de sonsonete y práctica carente de significado para el estudiante.

2. Discusión de la lectura, mediante formulación de preguntas que no contribuyen al desarrollo de unas destrezas de pensamiento más avanzadas, que están contempladas en el currículo actual.

3. Tendencia a traducir al español todo lo que el maestro dice en inglés e incluso el uso exclusivamente del español para presentar explicaciones y directrices a los estudiantes. Esta práctica no contribuye al desarrollo de destrezas de comprensión auditiva ya que el estudiante simplemente espera la explicación en español.

4. Uso exagerado de la lectura silenciosa en clase, en muchas ocasiones en sustitución de la lectura activa.

Por otra parte manifiesta que "el desarrollo de esta habilidad (la expresión oral) se ve limitada al contexto del aula y no a soluciones verdaderamente comunicativas dentro y fuera de ella". Esto indica que el profesor debe incentivar a los estudiantes con tareas extra clases para reforzar la temática impartida, quien oralmente tiene que practicar ejercicios, conversaciones, dialogar con sus compañeros, utilizando las frases aprendidas, escuchar las canciones y practicar con los amigos en varios contextos. González (2010) 
Para fomentar el desarrollo de la expresión oral, es necesario organizar el proceso de enseñanza aprendizaje, como un proceso que conduzca el profesor mediante su manera de proceder, los objetivos a lograr, la forma de practicar y evaluar con la atención a las actividades de los estudiantes que requieren del uso de la lengua como instrumento de comunicación con procedimientos cooperativos; así mismo, para lograr el desarrollo de las habilidades de expresión oral debe considerarse, los diferentes aspectos lingüísticos de forma integrada, porque al tratarse de forma aislada conduciría inevitablemente a un enfoque mecanicista.

El segundo momento de cambio ha producido otros escenarios de enseñanza aprendizaje. Como se conoce, la modalidad presencial es hoy en día compartida con otras formas que agilizan o contribuyen al logro de los objetivos propuestos en cualquier enseñanzaaprendizaje. En Ecuador, la enseñanza del inglés va implantándose con la modalidad tutorial como otra posibilidad de aprender el idioma en el cual los medios tecnológicos juegan un papel principal en el desarrollo de la autonomía para aprender.

La enseñanza- aprendizaje tutorial de forma más amplia abarca las vías de transmisión de los conocimientos a lo largo de generaciones en diferentes ámbitos, que incluyen el de la producción en pequeños talleres (enseñanza de un oficio a un aprendiz) hasta los más institucionalizados, como el caso de la enseñanza personalizada en la escuela (García Hoz, V. s/f). . A principios del siglo XX en Estados Unidos surge un proyecto educativo para la enseñanza básica denominado Plan Dalton, cuyas características fundamentales son la atención individualizada, la actividad educativa con la mayor autonomía posible para el estudiante, el autoaprendizaje, así como el desarrollo del sentido de la responsabilidad y la satisfacción por el trabajo. Éste es un proyecto exitoso que se conoce en otras partes del mundo como Inglaterra, Japón y los países nórdicos, lugares donde se desarrollan propuestas similares

En las instituciones de educación superior europeas y estadounidenses las tutorías tienen una larga tradición. En EEUU las tutorías sirven para dilucidar los retos de aprendizaje entre el aprendiz y el tutor en situaciones menos amenazadoras que el aula (R. Dunn and K Dunn 1993; Allen y Seaman, 2007; Morgan 2010). En Inglaterra, las tutorías constituyen la forma de enseñanza única para el doctorado; en España se han desarrollado de manera especial en la enseñanza secundaria (Rus, 1999) y en la educación superior es paradigmática la Universidad Nacional de Educación a Distancia (UNED) (Garcia , 1996). 
En las universidades mexicanas son un ejercicio académico reciente, los antecedentes más lejanos se ubican en el nivel de posgrado, donde la investigación se considera primordial y, por ello, la figura del profesor tutor resulta fundamental. En Cuba, hay antecedentes de tutorías a distancia mediante el uso de la tecnología, como la radio y la televisión, para niveles de enseñanza básicos y secundarios que le permitieron desarrollar una concepción del trabajo semipresencial en la enseñanza.

En Ecuador no existe una universidad pedagógica, o de otra índole, que establezca los lineamientos políticos y estratégicos de la formación docente impartida por las universidades, como acontece en Colombia y Venezuela, ni un entrenamiento para enseñar a ser profesor de la modalidad tutorial. Sin embargo, hay dos universidades que cuentan con una importante cantidad de programas formativos para docentes y tiene el mayor número de alumnos, al comparar con otras universidades: la Universidad Central de Ecuador, con su sede principal en la ciudad de Quito, y la Universidad Técnica Particular de Loja, que, como su nombre lo dice, tiene su sede principal en Loja, pero que al ser una oferta de educación a distancia, tiene muchos centros de referencia en varias ciudades de Ecuador y en otros países donde viven los ecuatorianos.

La modalidad tutorial implica el establecimiento de pares, tutor-alumno, en el proceso de enseñanza-aprendizaje. La modalidad tutorial garantiza la atención individualizada al estudiante en su proceso de aprendizaje, en este sentido el tutor facilita el aprendizaje cuando crea las condiciones necesarias para que el estudiante exprese sus potencialidades en la realización de tareas que promueven su desarrollo.

El estudiante asume una posición activa en su aprendizaje cuando desempeña con responsabilidad las tareas planteadas por el tutor, analiza y discute con él su planificación, ejecución y evaluación.

Durante toda la duración del curso el estudiante estará en contacto permanente con un tutor personal. Una persona que sabe lo que este necesita y que está dispuesta a compartir con él sus conocimientos. Podrá contar con su apoyo siempre que lo necesite -24 horas al día- para resolver sus dudas, orientarlo en su aprendizaje y evaluar su aprovechamiento del curso. Y detrás de él, toda una compleja estructura tutorial compuesta por expertos en cada materia apoyando su esfuerzo. Además, podrá intercambiar experiencias y conocimientos y contrastar opiniones con otros estudiantes de su curso y de otros cursos a través de, una auténtica 
Rev. SINAPSIS, Vol. 4, No 1, Junio 2014

ISSN $1390-7832$

comunidad virtual a cuyo foro de opinión puedes acceder siempre que lo desees. (CESDE, 2014)

Otro sentido de enseñanza tutorial está asociado a la competencia tutorial que ofrecen los juegos, en sentido general. Una de las principales dificultades que surgen al intentar precisar el concepto de tutoría se relaciona con la diversidad de significados en juegos, ligados a un campo conceptual amplio y denso que remite a figuras distintas de la tutoría y por lo mismo de sus funciones y prácticas (Sánchez, 2000).

La tutoría también es concebida como una forma de atención educativa donde el profesor apoya a un estudiante o a un grupo de estudiantes de una manera sistemática, por medio de la estructuración de objetivos, programas por áreas y técnicas de enseñanza apropiadas a la integración de grupos conforme a ciertos criterios y mecanismos de monitoreo y control, entre otros Alcántara (1990). En la tutoría se propicia una relación pedagógica diferente a la que establece la docencia ante grupos numerosos; en este caso el profesor asume el papel de consejero o "apoyo de aprendizaje"; el ejercicio de su autoridad se suaviza hasta casi desaparecer; el ambiente es mucho más relajado y amigable.

La Asociación Nacional de Universidades e Instituciones de Educación Superior, en su propuesta institucional de tutorías la define como un proceso de acompañamiento durante la formación de los estudiantes que se concreta mediante la atención personalizada a un grupo reducido de alumnos por parte de académicos competentes y formados para esta función. Es distinta y a la vez complementaria a la docencia frente a grupo, pero no la sustituye. ANUIES (2000) la Educación Superior del s. XXI. México

En el discurso actual sobre tutorías se ha generado un debate acerca del sentido del acompañamiento que ésta implica:

..la noción de acompañamiento parece haber emergido de las dificultades encontradas por los actores del campo educativo para responder a dos tipos de exigencia: la preocupación de un alumnado desorientado, considerado, no obstante, como ser autónomo o capaz de llegar a serlo, y la exhortación de resultados, de excelencia y de eficacia siempre más altos, provenientes de los responsables de la institución (Ghouali, 2007). 
Desde esta posición acompañar se define como un proceso que dinamiza tres lógicas: relacional, espacial y temporal. La relacional es concebida como una conexión, como "unirse a alguien"; la espacial se refiere a un desplazamiento, a "ir a donde él va"; y la temporal tiene que ver con estar con el otro al mismo tiempo (Ghouali, 2007).

Para Ardoino (2000) sólo se acompaña a alguien si esta persona sabe a dónde va. El acompañante es una persona activa, pero la acción es del acompañado. Este es un proceso de intersubjetividad "...en donde los sujetos se comunican en dos posiciones diferentes, donde el acompañante se pone en juego y acepta que el acompañado esté con él en una relación de alteración mutua. Donde el otro cambia al mismo tiempo que intenta cambiarlo. A través de esta idea, la dimensión ética del acompañado es subyacente" (Ardoino, 2000).

Desde otra perspectiva sociológica, ese acompañamiento se reconoce como la energía emocional explicada por Collins (2005) como fuerza motivadora principal en la vida social para el amor, odio, trabajo, consumo, culto, etc., la cual va desde los más altos grados de entusiasmo, seguridad en sí mismo, iniciativa hasta los más inferiores de apatía, depresión y arrepentimiento. Esta energía proviene de interacciones habituales que se asocian por el éxito o fracaso con lo que forman cadenas emocionales con otros en conversaciones, encuentros familiares, trabajos cotidianos, afiliaciones políticas y sociales entre otros patrones de relación social que la literatura aún no explica exhaustivamente el cómo aprovecharlas en la enseñanza y el aprendizaje, para los que la modalidad tutorial presenta amplios campos de indagación e investigación.

Para los autores de la presente investigación si se acepta que la vida social se encauza mediante la generación y distribución de energía emocional Collins (2005), también esa vida toma sentido direccionándola hacia lo que es cotidiano y estable en el individuo como sus hábitos, su uso del código lingüístico y la prioridad laboral pues son los elementos recurrentes en su vida y sobre lo que va a girar fundamentalmente el contenido comunicativo de la persona. Ese campo no puede desaprovecharse para ejercer influencias positivas que conduzcan a experiencias educativas del individuo en contextos informales, lo cual es un reto para la actual modalidad tutorial, incluso lo es más en el aprendizaje de una lengua extranjera la cual no siempre es inducida en esos contextos informales y cuyo efecto educativo se pierde en muchos casos.

Por otra parte, la tutoría puede estar relacionada al apoyo que el docente da al alumno en su formación para la toma de decisiones acertadas, recomendándole bibliografía o sólo 
ofreciendo opiniones sobre la vida académica de la institución; también puede ubicarse en relación con la formación de investigadores en un campo científico en particular. Para la tutoría realizada cuando se enseña a investigar al alumno la base de su accionar está en las funciones que se requieren de la acción y conducción del tutor y que se desarrolla con condiciones institucionales particulares apropiadas para lograr este propósito: la necesidad que tiene de uso en la institución y las condiciones en que se lleva a cabo. En esta práctica se requiere del compromiso del estudiante y el tutor, en su espacio académico y educativo lo que favorece lazos particulares en las relaciones diarias, con referencias contextuales según el profesor y el alumno, con la afectividad ligada a la información en la comunicación de ambos en cada situación de intercambio e interacción.

En el ámbito de la investigación es el tutor como investigador experto quien se responsabiliza académicamente del estudiante de manera inmediata, directa y permanente; establecen una relación de trabajo y crean un proyecto de formación específico para que el estudiante se convierta en investigador Sánchez (2000).

Este proceso tiene importancia porque el tutor transmite el conocimiento que necesita el alumno para hacerse investigador. Por eso, los autores de este trabajo concuerdan con Sánchez Puentes (2000) quien señala que el investigador tutor transmite tres tipos de saberes: los teóricos, los prácticos y los significativos con cuales se coincide con aquellos que se han estado expresando en este trabajo para la enseñanza aprendizaje del inglés y que por tanto se puede utilizar en ella desde la perspectiva que investigación también le ofrece al alumno.

a) Los saberes teóricos se refieren a las teorías y conocimientos reconocidos socialmente, paradigmas, supuestos teóricos y marcos conceptuales. Evidentemente su carácter va a ser conceptual.

b) Los saberes prácticos conforman el conjunto de estrategias, habilidades y destrezas propias del oficio del investigador. A través de este tipo de saberes se enseña y se aprende a problematizar, a fundamentar la teoría, a concebir la teoría como una herramienta de análisis, a organizar, analizar e interpretar la información. Cada uno de estos quehaceres de la investigación está integrado por numerosas y diferentes operaciones que requieren conocimientos teóricos, habilidades y estrategias. Se entiende pues el carácter procedimental de estos saberes. 
c) Los saberes significativos. El tutor también enseña a cuestionar acerca de los fines últimos de la investigación, los por qué de carácter ético-político del saber científico, así como los para quiénes de la producción científica. Se refiere a los significados y objetivos últimos del conocimiento que se genera. Así se confiere un carácter actitudinal a estos saberes.

Estos tres tipos de saberes insertos en las tradiciones disciplinarias, en procesos de socialización y circulación y en las posibilidades institucionales reales permean la formación de las nuevas generaciones de profesionales, pues es la forma en que la investigación se incluye en la práctica cotidiana y de la cual el profesor tutor y el estudiante tutorado se nutren para su desarrollo.

La utilización de modelos centrados en el alumno y la orientación hacia el aprendizaje por la vía de la tutoría académica requiere de la capacitación y la colaboración por parte de los distintos actores universitarios. Muchas veces, esta superación se propicia desde la actividad educativa presencial, por ello, están muy relacionadas las formas tutoriales y presenciales de educación. (J. Borges, 2009)

La Universidad Estatal del Sur de Manabí (UNESUM) pone énfasis en los estudios de necesidades de formación de recursos humanos en los rediseños curriculares de las carreras y actualiza los contenidos en relación con las competencias a desarrollar en ellos.

Implementar varias acciones a fin de continuar respondiendo a la demanda social a través de una oferta académica pertinente, renovada y actualizada que garantice una sólida formación profesional, científica, técnica y humanista es un objetivo vital de la educación. Para ello, la UNESUM completará el rediseño de la malla curricular de las carreras incorporando el sistema de créditos.

El tutor tiene la capacidad de ser no sólo un profesor, sino que proporciona, además y en paralelo la ayuda necesaria al estudiante. El tutor es el responsable de la formación integral del alumno desde todo ángulo de orientación educativa.

En la educación superior las relaciones tutoriales consisten en una estrategia de enseñanza aprendizaje, en que un experto conduce y orienta este proceso sobre la base de recrear situaciones de aprendizaje que se caracterizan por una gran independencia y autonomía del aprendiz, pero bajo la dirección y observación de un tutor. Como se ha venido reiterando la modalidad tutorial tiene como característica fundamental la conducción y orientación del alumno por una persona capaz de llevarlo a un estadio superior del conocimiento. 
Rev. SINAPSIS, Vol. 4, No 1, Junio 2014

ISSN $1390-7832$

El contenido de la tutoría estará dirigido esencialmente a la concreción de la estrategia educativa como respuesta a las principales necesidades de los estudiantes, identificadas en su diagnóstico, caracterización y evaluación. Lo cual es importante también en el contexto ecuatoriano. Así se define la modalidad tutorial como la más flexible en cuanto a su organización, contenido y vías de control, lo cual requiere de precisión individual desde el diagnóstico hasta el logro del objetivo planteado, lo cual debe caracterizar el accionar metodológico si se usa en el aprendizaje de la lengua inglesa con fines formativos profesionales.

Hoy día se abren nuevas consideraciones y surgen nuevas experiencias acerca del tutor y su papel en la atención al estudiante de la educación superior. Así se está tratando el tema desde diferentes enfoques y perspectivas: desde el desempeño profesional pedagógico del tutor en la escuela primaria, desde el papel en la producción intelectual y desde la formación de otros como orientador y guía en los campus universitarios, entre otros muchos más. Todas estas aproximaciones indican un reconocimiento al papel del tutor y de la tutoría como estrategia para elevar la calidad de la educación, lo que conduce además a la necesidad de reflexión sobre los objetivos de la modalidad presencial y la tutorial y cómo se complementan en la enseñanza y el aprendizaje de idiomas.

Considerando entonces estos aspectos generales que advierten la importancia de la tutoría y su repercusión en el desarrollo cultural del estudiante de diferentes carreras desde el inglés, es necesario implementar vías concretas que permitan su óptimo desarrollo en la formación del profesional.

Al tutor como se ha expresado se le ha asignado diferentes roles según la posición que se asume y no se ha visto como un conjunto de ellos que inexorablemente han de cumplirse en el proceso de enseñanza aprendizaje paralelamente al rol del profesor, porque el estudiante seguirá siendo uno en su formación profesional y necesita que las influencias converjan. Al tutor le han dado casi siempre la misión de consultante del alumno. Esa posición refuerza la distancia entre profesores y estudiantes, y se refiere a la actividad tutorial como intermediario. Sin embargo, su papel es importante también en aquellos sistemas a distancia que no posean medios modernos para la consulta a distancia, y en ellos debe también aportar vías de satisfacción espiritual y apoyo al que aprende como mediador del desarrollo regulador emocional de esos aprendices por los medios telemáticos. El tutor aunque en un ambiente 
Rev. SINAPSIS, Vol. 4, No 1, Junio 2014

ISSN $1390-7832$

virtual debe aprovechar los recursos tecnológicos para acompañar y ofrecer energía emocional que permitan el aprendizaje de los estudiantes.

Esta misión del tutor se adecua a lo expresado por Vigotsky (1978) en cuanto al apoyo de los coetáneos en el desarrollo de la Zona de Desarrollo Próximo. Zona de desarrollo que se hace necesaria amplificar en la formación y desarrollo de competencias en un idioma extranjero, el cual se convierte en un vehículo para la comunicación del futuro profesional que se está formando.

Es indispensable que el estudiante sienta la necesidad de aprender el inglés, porque le sirve para la vida y no sólo, porque le es necesario aprobar la asignatura para pasar de nivel. Pero no basta con que sólo desde esta asignatura sea resaltada su importancia, es pertinente que las restantes asignaturas del currículo contribuyan a ello, y que desde esa interacción con las demás, se encuentre la relación entre aquellos elementos del contenido de aprendizaje y su mundo afectivo-motivacional, o lo que es igual, que alcance sentido para su vida.

Fuentes especializadas acerca del proceso de enseñanza y aprendizaje del inglés, de una manera u otra sustentan el valor del protagonismo del estudiante en dicho proceso, y en muchos casos ofrecen vías y procedimientos para su materialización "el proceso del pensamiento es igual en cualquier lengua..." Antich (1988) y otros. Indiscutiblemente, lograr la autonomía del aprendizaje del alumno es un objetivo deseado que se va canalizando mediante la comunicación y las necesidades crecientes del estudiante en este proceso.

Esa autonomía mediante el proceso comunicativo en lenguas extranjeras es necesidad del profesional y es un componente integrante de la formación por la profesión que abarca mucho más que el momento formal del aula. La propia motivación del alumno hace que su comunicación sea formativa en otros contextos informales y en situaciones sin relaciones aparentes con su profesión, por eso se concuerda con Borges (2006) que: “... la motivación profesional se caracteriza por la existencia de formaciones motivacionales diversas que participan en la regulación de la actividad profesional, en cuya base predominan motivos relacionados con el desempeño profesional. Los sujetos que muestran estas necesidades expresan su orientación a partir del planteamiento de objetivos relacionados con el contenido de la profesión misma, a la vez que expresan vivencias afectivas positivas en su consecución..." Este aprendizaje potencial en contextos informales a partir de la propia motivación del sujeto paralelo al que se logra en contextos formales y que lo complementa, es el que en esta investigación se vislumbra como un vacío teórico que se debe completar para 
poder explicar metodológicamente desde una modalidad diferente y actual el proceso de enseñanza aprendizaje del inglés en la formación del profesional de Ingeniería Civil y otras ramas técnicas en las actuales circunstancias tecnológicas.

La vida moderna implica la tecnología en cada acción que se acomete. Actualmente la existencia de la tarifa plana, que ofrecen las compañías telefónicas de casi todos los países, y el acceso gratuito a Internet en una banda, ha provocado el incremento de su utilización, en detrimento de otras vías de comunicación más convencionales, como la prensa, el correo postal, el teléfono y hasta la comunicación oral en las aulas. Además, las telecomunicaciones se perfeccionan cada día y permiten ya la consulta de documentos videográficos y sonoros, como videos a la carta, participación en debates y concursos, y entornos lúdicos y educativos, entre otros. Todos estos podrían también facilitar el desarrollo del código oral de los estudiantes y su implicación activa en el aprendizaje de una lengua extranjera en la universidad.

De allí que han surgido la Technology enhanced language learning (TELL), la Enseñanza de lenguas asistida por computadoras (ELAC) y la peninsular Enseñanza de lenguas asistida por ordenador (ELAO), todas ellas dirigidas a valorar el efecto en la enseñanza con el uso de la tecnología. Aunque ninguno de sus estudios explican la relación entre la motivación del sujeto por su fin de formación profesional que lo oriente a complementar los contextos formales e informales del aprendizaje de una lengua extranjera con el control presencial y tutorial del docente para incrementar el desarrollo de la competencia comunicativa oral de esa lengua desde una modalidad que combine lo presencial y lo tutorial.

El uso de internet, como medio derivado del incremento tecnológico y medio educativo, ha facilitado mucho más el código escrito que el oral entre los usuarios, quienes inconscientemente usan un idioma o varios para su comunicación cotidiana. Este medio por tanto tiene un inconveniente para desarrollar la habilidad de escuchar y la de hablar. De allí que en su implementación para la enseñanza hay que aprovechar todo lo que se ha explicado sobre el empleo de métodos tutoriales que desarrollen la habilidad de escuchar y la de expresión oral.

\section{Conclusiones}

En correspondencia con lo expuesto el profesor debe ofrecer la adecuada atención al estudiante a fin que este eleve su rol protagónico en el aprendizaje del inglés desde lo presencial y tutorial. Este aspecto encuentra justificación en los referentes teóricos analizados, 
Rev. SINAPSIS, Vol. 4, No 1, Junio 2014

ISSN $1390-7832$

porque en ellos se argumenta la importancia del protagonismo del estudiante como indicador de calidad del aprendizaje para realizar propuestas en la enseñanza y el aprendizaje.

Es preciso garantizar la formación y desarrollo de actitudes y valores, y la apropiación de conocimientos y habilidades, comunes y específicos del estudiante, desde el Inglés, como expresión de un enfoque interdisciplinario. Pero también sobre la base de aprovechar las potencialidades que los contextos informales posibilitan en la formación de la cultura general de los estudiantes, porque el proceso de enseñanza aprendizaje debe ofrecer más oportunidades metodológicas que conduzcan a fortalecer el desarrollo de la competencia comunicativa oral hacia un acercamiento efectivo entre las actividades del aula y su hogar, de forma consensuada, a través de la modalidad combinada de enseñanza aprendizaje presencial y virtual, de la lengua inglesa, con sustentación tecnológica, en la carrera de Ingeniería Civil.

\section{Bibliográfica}

1. Addine Fernández, F.; A. M. González Soca, y S. Recarey Fernández (2004) Didáctica. Teoría y práctica. La Habana: Ed: Pueblo y Educación.

2. Addine, F. (2000). y otros. Diseño Curricular. Instituto Pedagógico Latinoamericano y Caribeño, La Habana, (material en soporte electrónico)

3. Aguerrondo, Inés (2009). Conocimiento complejo y competencias educativas Ginebra: UNESCO Oficina Internacional de Educación.

4. Alcántara, A. (1990). "Consideraciones sobre la tutoría en la docencia universitaria" Perfiles Educativos, No. 49-50: 51-55

5. Ballesteros Ma, Alfonso (2010). Alternativa metodológica para la formación de la cultura artística desde la disciplina inglés en la carrera de Medicina [tesis doctoral]. Camagüey: Centro de Estudio de la Ciencia de la Educación, Universidad de Camagüey Ignacio Agramonte y Loynaz.

6. Allen, E. I. and Seaman. (2007); Blending in. The Extent and Promise of Blended Education in the United States.

7. Allende, M. (2011). Comunicación: regla de Mehrabian. http://intervencioneseficaces.blogspot.com/2011/11/comunicacion-regla-demehrabian.html Retrieved 17 febrero 2013.

8. Alvarez DE Zayas, C.M. (1999). La escuela en la vida. Didáctica. Ciudad de la Habana: Editorial Pueblo y Educación. 
9. Antich de Leon, R. y otros (1986). Metodología de la Enseñanza de lenguas extranjeras. Ciudad de La Habana: Editorial Pueblo y Educación.

10. Ardonio, J. (2000). Les avatars de l'éducation, París: PUF.

11. Asensio, M; Carretero, M; Pozo, J. (1986). La comprensión de la historia. Pensamiento relativista. Cuadernos de Pedagogía 133: 24- 27.

12. Borges, J. L. (2006). Modelo de gestión didáctica del post grado a distancia. Tesis de doctorado. Universidad de Oriente. Santiago de Cuba.

13. Boud, David. Ruth Cohen, Jane Sampson (2001). Peer Learning in Higher Education: Learning from \& with Each Other. Sidney, Australia: Routledge.

14. Brown, H. D. (2000). Principles of Language Learning and Teaching. 4th Edition. New York: Person Education Company.

15. Brown, H. D. (2009). Teaching by Principles. An Interactive Approach to Language Pedagogy. New Jersey: Prentice Hall Regents.

16. Brubacker, M. et al. (1999) Perspectives in Small Group Learning. Canada: Rubicon Publishing Inc.

17. Castellanos Simons, D. y otros (2005). Aprender y enseñar en la escuela: Una concepción desarrolladora. Ciudad de la Habana: Ed. Pueblo y Educación.

18. Castellanos, D. (2002). y otros. Aprender y enseñar en la escuela: una concepción desarrolladora. ISPEJV, La Habana, (versión digital).

19. Cebrián, J.L. (1998). La red. Cómo cambiarían nuestras vidas los medios de comunicación. Círculo de lectores. Barcelona.

20. Cesde. (2014). Centro de Estudios Superiores de Empresa. Curso de Especialista en Técnicas Tutoriales para el E-learning que dinamizará tu carrera profesional. http://www.tumaster.com/Cursos:-Curso-de-Tecnicas-de-Tutoriales-para-el-Elearningmmasinfo19754.htm. Retrieved 15 -5- 2014

21. Collins, R. (2005). Interaction Ritual Chains. Princeton University Press.

22. Dobson, J. M. (1992). Effective Techniques for English conversation groups. New York: Newbury House Publisher.

23. Dunn, R. and Dunn K (1993) . Teaching Secondary Students through Their Individual Learning Styles: Practical Approaches for Grades 7-12.Boston: Allyn \& Bacon .

24. Ferraté Pascual, G. (s/f) Enseñanza Internet y educación Universitat Oberta de Catalunya. Educaweb 
25. García Aretio, L. (1996). "La educación a distancia: Concepto y componentes. Programa de superación académica. Educación no escolarizada”. UAM, México D.F: Revista Iboeroamericana de Educación a Distancia. Volumen 1: 2.

26. García Esteban, S. (2013) Análisis y evaluación de cursos de inglés de los negocios a distancia asistido por las nuevas tecnologías de la información y la comunicación. Tesis doctoral. Universidad de Alcalá.

27. García Hoz, V. Diccionario Pedagógico Labor, s/f. http://www.recoleta.edu.pe/biblioteca/catalogo/ver.php?id=7319. Retrieved May 23, 2014.

28. Ghouali, H. (2007). "El acompañamiento escolar y educativo en Francia. Revista mexicana de investigación educativa". Vol. 12, núm. 32: 207-242.

29. González, F. (2004). Acerca de la Metacognición. Universidad Pedagógica Experimental Libertador, Venezuela. http://cidipmar.fundacite.org.ve/doc/Paradigma

30. González. Y. ( 2010) Ejercicios para desarrollar habilidades de expresión oral en idioma inglés. Facultad de Cultura Física Santi Spiritus. http://www.monografias.com/trabajos37/expresion-oral-ingles/expresion-oralingles2.shtml\#ixzz36RZRotof

31. Hamer, J. (2008). The Practice of English Language Teaching.3rd Edition. UK: Longman.

32. Latapi Sarre, P. Investigador Nacional. La Enseñanza Tutorial: Elementos para una propuesta orientada a elevar la actividad.

33. Martínez LLantada, M. (2007). y otros. Inteligencia, creatividad y talento: debate actual. La Habana: Editorial Pueblo y Educación

34. Morgan, H. (2010). Improving Schooling for Cultural Minorities: The Right Teaching Styles Can Make a Big Difference. Educational Horizons.

35. Rodríguez Díaz, O. (2008). Metodología integradora para el desarrollo de la competencia metalingüística profesional en los estudiantes de la carrera Licenciatura en Educación, Especialidad Inglés. Tesis en opción al título de doctor en Ciencias Pedagógicas. ISP José Martí, Camagüey.

36. Rus, A. (1999): Tutoría, departamentos de orientación y equipos de apoyo. Granada: Universidad de Granada.

37. Sánchez, R. (2000). La arquitectónica de la investigación educativa, en Enseñar a investigar. Una didáctica nueva de la investigación en ciencias sociales y humanas. México: Cesu-Unam, pp. 127-155. 
Rev. SINAPSIS, Vol. 4, No 1, Junio 2014

38. Silva Ros, M. (2006) La enseñanza del inglés como lengua extranjera en la titulación de filología inglesa: el uso de canciones de música popular no sexista como recurso didáctico. http://www.biblioteca.uma.es/bbldoc/tesisuma/16853805.pdf tesis de doctoral. Retrieved: enero, 13, 2014.

39. Silvestre Oramas, M. y P. Rico (2003). Compendio de Pedagogía. Ciudad de la Habana: Ed. Pueblo y Educación.

40. Valenzuela Peñafiel, R. (2007). Autoevaluación institucional con fines de acreditación. Escuela Politécnica Nacional. Quito.

41. Vigotski, L. S. (1978). El Desarrollo de Los Procesos Psicológicos Superiores. Barcelona: Grijalbo. 